\title{
Drift-Diffusion Simulations of Potassium Channels
}

\author{
Jeremiah Jones \\ Arizona State University \\ jrjones8@asu.edu \\ Sponsor: Dr. Carl Gardner
}

April 26, 2010

\begin{abstract}
Ionic channels play an important role in regulating the cell's membrane potential and internal charge. This paper will focus on a continuum model of the KcsA potassium channel. We will derive the Poisson-Nernst-Planck (PNP) equations in general and then provide computational solutions for a 1D KcsA channel using experimentally determined parameters. The solution to the PNP equations consists of the time-dependent charge densities of each ion, coupled with the electric potential. The results will be used to solve for the time-dependent current in the channel in response to different time-dependent voltage signals, which can be compared with experimental data.
\end{abstract}




\section{Introduction}

\subsection{Ionic Channels}

Cellular membranes are one of nature's many amazing inventions that make life possible. The membrane separates the internal components of a cell from its surrounding environment and controls traffic into and out of the cell. Membrane thicknesses are usually on the order of a few nm-four orders of magnitude smaller than a typical eukaryotic cellular radius. Their physical and chemical structures are incredibly complex, consisting of a mosaic of proteins and lipids that are involved in a wide variety of the cell's activities. Among these activities are controlling the concentration of various ionic species, which in turn affects the electric potential across the membrane. Complex protein structures in the membrane form ionic channels, which can be thought of as holes in the membrane that allow ions to pass through them. A typical cell has on the order of $10^{4}$ such channels that vary widely in function and complexity. Although some channels allow the passage of ions based solely on charge, we will be investigating the KcsA potassium channel, which is selective, i.e., it only allows one kind of ion $\left(\mathrm{K}^{+}\right)$to pass though. The KcsA channel is found only in bacteria but it makes an ideal model for studying channels since its complete structure has been determined via X-ray crystallography. Ionic channels also have many different gating mechanisms that trigger the channel to open or close. However, in this paper, we will assume that the channel is already open and will make no assumptions about the gating mechanism of the channel.

The existence of ionic channels has been known for quite some time and they continue to be an active area of research in cellular biology. These channels play an important role in many biological processes such as chemical signalling in the endocrine system as well as electrical signalling in the nervous system. Understanding their properties and the mechanisms by which they operate has yielded valuable insights into many biological phenomena. Many heart-related illnesses are treated by drugs that are designed to either prolong or accelerate the process of repolarization due to large electrical impulses by manipulating the channels. Experimental methods for examining channels, such as patch-clamp techniques, have been employed by biologists since the 1960's, but they are limited by the amount and quality of data that they can provide. However, we can study many channel properties and make useful predictions by way of numerical simulations based on simplified mathematical models. For a thorough study on the biology of ionic channels, see [1]. 


\subsection{The PNP Model}

In the following discussion, we will derive the equations used to model ionic channels. We begin by considering a channel that is surrounded by intracellular and extracellular salt baths (water with dissociated ions) as shown in Figure 1. We will assume that the baths contain only potassium $\left(\mathrm{K}^{+}\right)$and chloride $\left(\mathrm{Cl}^{-}\right)$ions as these are the ions that play the greatest role in KcsA channels. The channel also carries a fixed background charge due to ions embedded in the membrane protein, which is overall negative.

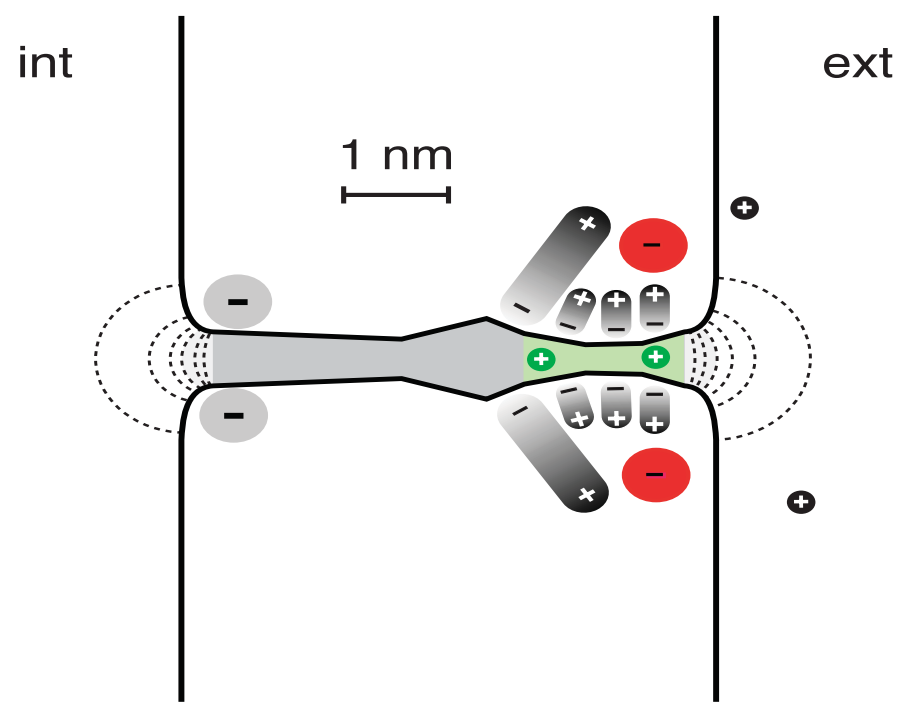

Figure 1: Simple schematic of the $\mathrm{K}$ channel. The green charges represent the $\mathrm{K}^{+}$ ions travelling through the channel and the black charges represent the $\mathrm{K}^{+}$ions in the extracellular bath. Gray and red charges represent the fixed background charge of the channel. The dashed curves at the ends of the channel represent equipotential surfaces.

To simulate the behaviour of the ions in the channel, we consider an open channel with a voltage bias $V$ across the membrane. Our goal is then to determine the time-dependent distribution of ions in the baths and channel. A well-known model known as the Poisson-Nernst-Planck (PNP) model (or drift-diffusion model) allows us to analyze this system by treating the ions as a continuum, instead of discrete objects. The validity and accuracy of the PNP model has been verified by the work done in [4]-[6]. This model uses two physical heuristics to determine a 
system of partial differential equations to model this scenario. The first heuristic relies on the fact that there is a local conservation law for each ionic species. This is stated mathematically as

$$
\frac{\partial n_{i}}{\partial t}+\nabla \cdot \mathbf{J}_{i}=0, \quad i=\mathrm{K}^{+}, \mathrm{Cl}^{-}, \mathrm{Na}^{+}, \text {etc. }
$$

where $n_{i}(\mathbf{x}, t)$ is the number density (ions/volume) and $\mathbf{J}_{i}(\mathbf{x}, t)$ is the current density of ionic species $i$. The second heuristic comes from Gauss's Law, which relates the electric potential in a given region to the charge density contained in that region. The mathematical formulation of this statement is

$$
\nabla \cdot(\epsilon \nabla \phi)=-\rho
$$

where $\epsilon(\mathbf{x})$ is the dielectric coefficient of the medium (bath or channel), $\phi(\mathbf{x}, t)$ is the electric potential and $\rho(\mathbf{x}, t)$ is the total charge density. Equations (1) and (2) form the general PNP model. Note that these equations are not independentthey are implicitly coupled because the current density depends on the potential and the potential depends on the ion densities. To apply this model to a specific channel, we must first find expressions for the current densities $\mathbf{J}_{i}$ and the total charge density $\rho$.

Current is defined as the movement of charge, so to find an expression for the current density we must consider what makes the ions move. There are two such mechanisms: concentration gradients and potential gradients. Thus, we should expect two terms in the current density, one to represent each gradient. Moreover, the concentration gradient of species $i$ should be proportional to $\nabla n_{i}$ and the potential gradient flux should be proportional to both $n_{i}$ and $\nabla \phi$. The full expression for the current density of ionic species $i$ is thus

$$
\mathbf{J}_{i}=-D_{i} \nabla n_{i}-z_{i} \mu_{i} n_{i} \nabla \phi
$$

where $D_{i}(\mathbf{x})$ is the diffusion coefficient, $z_{i}$ is the valence and $\mu_{i}$ is the mobility coefficient. The coefficients $D_{i}$ and $\mu_{i}$ measure the effectiveness with which the concentration gradient and the potential gradient, respectively, move a particular ion. Determining the total charge density in the region of interest is much simpler: it is just the sum of the charges of the ions in the salt baths plus the fixed background charge embedded in the channel protein. Thus,

$$
\rho=-e N+e \sum_{i} z_{i} n_{i}
$$

where $e$ is the unit charge and $N(\mathbf{x})$ is the number density of the background charge. Combining equations (3) and (4) with (1) and (2), we have 


$$
\begin{gathered}
\frac{\partial n_{i}}{\partial t}=\nabla \cdot\left(D_{i} \nabla n_{i}+z_{i} \mu_{i} n_{i} \nabla \phi\right) \\
\nabla \cdot(\epsilon \nabla \phi)=e N-e \sum_{i} z_{i} n_{i} .
\end{gathered}
$$

This system of equations represents the general PNP model and is valid for any ionic channel.

\section{Implementation}

We now form a well-posed problem by considering a one-dimensional KcsA channel with a fixed negative background charge $N(\mathbf{x})$ that is surrounded by intracellular and extracellular salt baths. Figure 2 shows a schematic of the $1 \mathrm{D}$ region along with boundary conditions, which will be discussed in the next subsection. The length of the channel $(3.5 \mathrm{~nm})$ is known from experimental observation. The choice of extending the bath by $5 \mathrm{~nm}$ on each side of the channel seems somewhat arbitrary but it has been observed that this is approximately the distance over which the charge densities reach their asymptotic bath values. The baths will

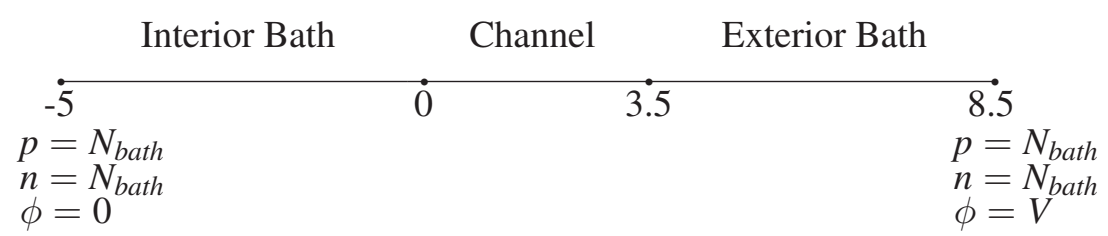

Figure 2: Computational 1D region (nm scale).

be considered to be homogeneous solutions of $\mathrm{K}^{+}$and $\mathrm{Cl}^{-}$ions in water with each species having an equal number of ions in the baths. This assumption guarantees that no current is flowing before the voltage is applied. At $t=0$, we apply a voltage bias $V$ across the membrane and solve for the time-dependent ion densities and potential in the region shown in Figure 2. In the following calculations, the densities of the $\mathrm{K}^{+}$and $\mathrm{Cl}^{-}$ions will be denoted as $p$ and $n$, respectively. The general PNP equations from the last section can now be applied to the system we are considering: 


$$
\begin{aligned}
& \frac{\partial p}{\partial t}=\frac{\partial}{\partial x}\left(D \frac{\partial p}{\partial x}+\mu p \frac{\partial \phi}{\partial x}\right), x \in[-5,8.5], t>0 \\
& \frac{\partial n}{\partial t}=\frac{\partial}{\partial x}\left(D \frac{\partial n}{\partial x}-\mu n \frac{\partial \phi}{\partial x}\right), x \in[-5,8.5], t>0 \\
& \frac{\partial}{\partial x}\left(\epsilon \frac{\partial \phi}{\partial x}\right)=e(N-p+n), \quad x \in[-5,8.5], t>0 .
\end{aligned}
$$

This system contains three state variables, $p, n$ and $\phi$, along with four parameters, $D, \mu, \epsilon$ and $N$. Note that the subscripts on $D$ and $\mu$ have been dropped. These parameters are specific to a given ionic species but they are determined by the mass and charge magnitude of the species. Since $\mathrm{K}^{+}$and $\mathrm{Cl}^{-}$are equal in charge magnitude and have a negligible difference in mass, we can assume that $D_{\mathrm{K}^{+}}=D_{\mathrm{Cl}^{-}} \equiv D$ and $\mu_{\mathrm{K}^{+}}=\mu_{\mathrm{Cl}^{-}} \equiv \mu$. In order to solve the given equations for the 1D channel, we must make use of experimentally determined values for the four parameters. All of these parameters are assumed to be piecewise constant functions of $x$ and their values in each region are given in Table 1. Note that the values in Table 1 have been scaled appropriately to be consistent with our computational units.

\begin{tabular}{|c|c|c|c|c|}
\hline Region & $D$ & $\epsilon$ & $\mu$ & $N$ \\
\hline$[-5,0)$ & 1.5 & 80 & 60 & 0 \\
\hline$[0,0.2)$ & 0.40 & 80 & 16 & 25 \\
\hline$[0.2,1.3)$ & 0.40 & 4.0 & 16 & 0 \\
\hline$[1.3,2.3)$ & 0.40 & 30 & 16 & 0.64 \\
\hline$[2.3,3.5)$ & 0.40 & 30 & 16 & 1.6 \\
\hline$(3.5,8.5]$ & 1.5 & 80 & 60 & 0 \\
\hline
\end{tabular}

Table 1: K channel parameters taken from data given in [2]. Lengths are given in $\mathrm{nm} ; D$ is given in $10^{-5} \mathrm{~cm}^{2} / \mathrm{s}$; $\epsilon$ is dimensionless in cgs units; $\mu$ is in $10^{-5} \mathrm{~cm}^{2} /(\mathrm{V} \mathrm{s})$; and $N$ is in $10^{21} \mathrm{~cm}^{-3}$. 


\subsection{Initial and Boundary Conditions}

For the initial conditions for equations (7) and (8), we assume that the entire region $[-5,8.5]$ begins with a uniform constant density $N_{b a t h}=0.1$ for each ionic species. However, we must also take into account the background charge in the channel. Thus we have

$$
p(x, 0)=N+N_{b a t h} \text { and } n(x, 0)=N_{b a t h} .
$$

We also have the ambient Dirichlet boundary conditions for equations (7) and (8) at the far ends of the baths:

$$
p(-5, t)=p(8.5, t)=n(-5, t)=n(8.5, t)=N_{b a t h}
$$

The boundary conditions for equation (9) are imposed:

$$
\phi(-5, t)=0 \text { and } \phi(8.5, t)=V(t)
$$

\subsection{Spatial Discretization}

To solve equations (7)-(9) numerically, we implement a finite difference scheme. This is a rather natural choice for the spatial discretization, given that the computational region is a line segment. While many PDE software packages are capable of solving these equations, the code for the following implementation was written by the author in MATLAB. The key reason for this is to begin developing the software that will be required to solve these equations on complex 2D regions, which would not be easily implemented using a PDE package.

We begin by defining all spatial functions on $M+1$ equally spaced grid points over the interval $[-5,8.5]$ with a spacing of $h=1 / M$. The grid points are labeled $j=0,1,2, \ldots, M$ with $j=0$ and $j=M$ representing the boundary points. All computations are thus performed on grid points $1,2, \ldots, M-1$ which couple to the boundary points. In this scheme, all spatial functions ( $p, n, \phi, N, \epsilon, \mu$ and $D)$ are represented as vectors of length $M-1$. Our goal is to transform the PDEs (7)(9) into matrix equations that can be solved computationally. Using second-order central difference approximations for the spatial derivatives, we the discretized 
versions of equations (7)-(9) at an arbitrary grid point $j \in\{1,2, \ldots, M-1\}$ are

$$
\begin{aligned}
&\left.\frac{\partial p}{\partial t}\right|_{j}=\frac{1}{h^{2}}\left(D_{j+\frac{1}{2}}\left(p_{j+1}-p_{j}\right)-D_{j-\frac{1}{2}}\left(p_{j}-p_{j-1}\right)\right) \\
&+\frac{1}{h^{2}}\left(\mu_{j+\frac{1}{2}} p_{j+\frac{1}{2}}\left(\phi_{j+1}-\phi_{j}\right)-\mu_{j-\frac{1}{2}} p_{j-\frac{1}{2}}\left(\phi_{j}-\phi_{j-1}\right)\right) \\
&\left.\frac{\partial n}{\partial t}\right|_{j}=\frac{1}{h^{2}}\left(D_{j+\frac{1}{2}}\left(n_{j+1}-n_{j}\right)-D_{j-\frac{1}{2}}\left(n_{j}-n_{j-1}\right)\right) \\
& \quad-\frac{1}{h^{2}}\left(\mu_{j+\frac{1}{2}} n_{j+\frac{1}{2}}\left(\phi_{j+1}-\phi_{j}\right)-\mu_{j-\frac{1}{2}} n_{j-\frac{1}{2}}\left(\phi_{j}-\phi_{j-1}\right)\right) \\
& \frac{1}{h^{2}}\left(\epsilon_{j+\frac{1}{2}}\left(\phi_{j+1}-\phi_{j}\right)-\epsilon_{j-\frac{1}{2}}\left(\phi_{j}-\phi_{j-1}\right)\right)=e\left(N_{j}-p_{j}+n_{j}\right) .
\end{aligned}
$$

Note that the subscripts $j-\frac{1}{2}$ and $j+\frac{1}{2}$ represent the values of the functions at midpoints, which are defined by linear interpolation. For example, $p_{j+\frac{1}{2}}=$ $\frac{1}{2}\left(p_{j}+p_{j+1}\right)$ and $p_{j-\frac{1}{2}}=\frac{1}{2}\left(p_{j-1}+p_{j}\right)$.

We can write equations (10)-(12) as linear systems of the form

$$
\begin{gathered}
\frac{\partial p}{\partial t}=A p+b_{p} \\
\frac{\partial n}{\partial t}=B n+b_{n} \\
C \phi=e(N-p+n)
\end{gathered}
$$

where $A, B$ and $C$ are tridiagonal difference matrices, $p, n$ and $\phi$ are the solution vectors and $b_{p}$ and $b_{n}$ are vectors containing boundary data for $p$ and $n$, respectively. This spatial discretization scheme effectively transforms equations (10)-(12) into a system of ODE's in time.

\subsection{Temporal Discretization}

An observant reader may notice that in order to calculate the matrices $A$ and $B$, it is necessary to first know $\phi$. Thus, a fully implicit method of solving equations (10)-(12) would require us to solve for $p, n$ and $\phi$ simultaneously. However, equations (10) and (11) contain nonlinear terms, which would require us to use Newton's method (or some other nonlinear solver). In order to avoid solving the nonlinear equations, we linearize the system by implementing a semi-implicit 
method which solves for $\phi$ using current data and then solves for $p$ and $n$. Although this method is not fully implicit, we can avoid numerical instabilities and arrive at a reasonable solution by taking moderately sized time steps. To solve equations (13) and (14), we must implement a temporal discretization scheme that is numerically stable, accurate and computationally fast. One of the best schemes that satisfies these requirements is the TRBDF2 method, which is L-stable and second-order accurate. This method will be briefly reviewed here but the reader is referred to [4] for a complete discussion.

Consider the ODE $\dot{u}=f(u)$ with $u(0)=u_{0}$. In the following discussion, $u^{k}$ will be used to denote $u\left(t_{k}\right)$. To compute $u^{k+1}$ from $u^{k}$, we first use the Trapezoid Rule (TR) to compute $u^{k+\gamma}$ :

$$
u^{k+\gamma}=u^{k}+\frac{\gamma \Delta t_{k}}{2}\left(f^{k}+f^{k+\gamma}\right) .
$$

We then use the second-order Backward Difference Formula (BDF2) to compute $u^{k+1}$ :

$$
u^{k+1}=\frac{1-\gamma}{2-\gamma} \Delta t_{k} f^{k+1}+\frac{1}{\gamma(2-\gamma)} u^{k+\gamma}-\frac{(1-\gamma)^{2}}{\gamma(2-\gamma)} u^{k} .
$$

The constant $\gamma=2-\sqrt{2}$ is designed to minimize the norm of the local truncation error at every time step. Notice that $\Delta t$ has a subscript $k$. This is to signify that $\Delta t$ is not constant, but is adjusted at every time step to optimize the computation time. To implement this dynamic time step, we add a subroutine to the program that computes the optimal $\Delta t$ within some window $\left[\Delta t_{\min }, \Delta t_{\max }\right]$ by monitoring a divided-difference estimation for the local truncation error (LTE). The LTE at time step $k+1$ is $\mathrm{LTE}^{k+1}=\alpha \Delta t_{k}^{3} u^{(3)}$, which can be estimated as

$$
\mathrm{LTE}^{k+1} \approx 2 \alpha \Delta t_{k}\left(\frac{1}{\gamma} f^{k}-\frac{1}{\gamma(1-\gamma)} f^{k+\gamma}+\frac{1}{1-\gamma} f^{k+1}\right),
$$

where

$$
\alpha=\frac{-3 \gamma^{2}+4 \gamma-2}{12(2-\gamma)}
$$

\section{Results}

Using the equations described in the previous sections, several numerical simulations using different spatial resolutions and final times were performed in MATLAB. Figures 3 and 4 show plots of the steady state densities and potential, respectively, with $V(t)=0.1$. These plots were generated using 540 spatial grid 
points and were simulated for $10 \mathrm{~ns}$, which was found (by numerical simulation) to be sufficient time for the channel to reach steady state. Note that the ordinate of Figure 3 uses a log scale. Also, the background charge $N$ is plotted with a dashed line for reference. Figure 3 shows significant boundary layers of charge near the

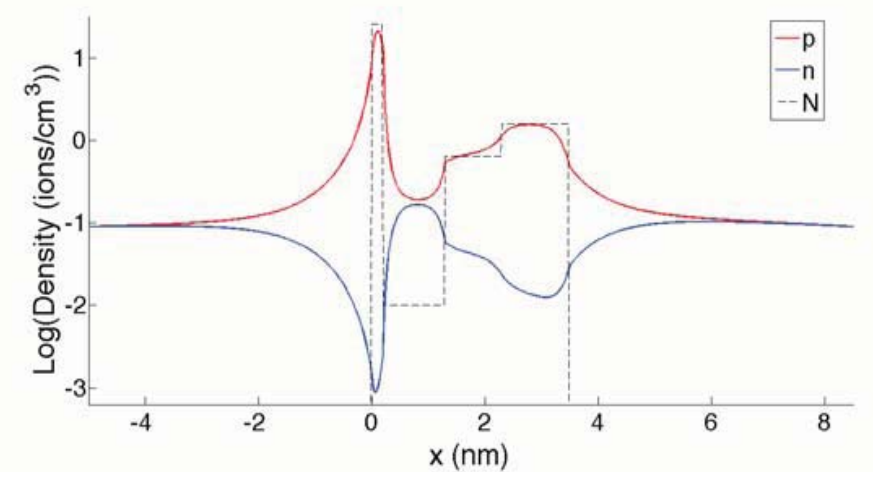

Figure 3: Steady state densities.

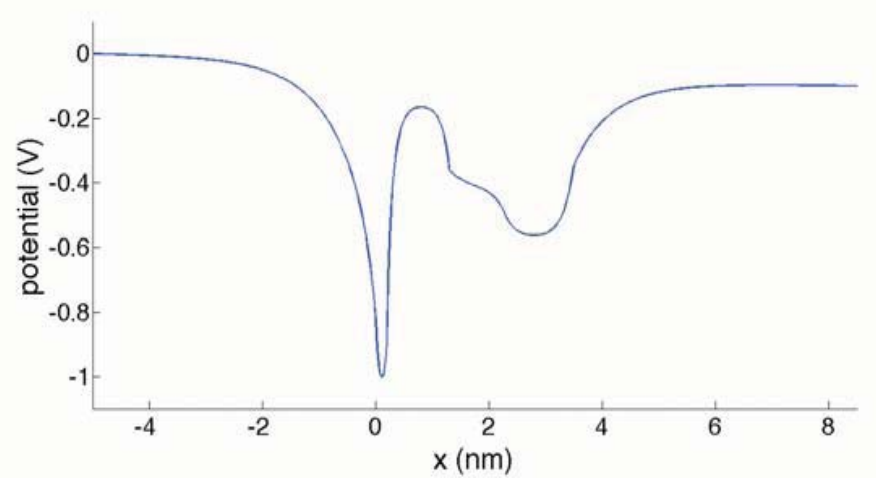

Figure 4: Steady state potential.

boundaries of the channel, suggesting that during steady state most of the charge has accumulated at the interface of the channel with the baths. Also, notice that $p$ roughly follows $N$ inside the channel. This implies that the channel is being driven toward electrical neutrality given that the sum of $N_{\text {chan }}$ and $n$ nearly cancel out $p$. Figure 3 also asserts that approximately $0.07 \mathrm{Cl}^{-}$ions are present in the channel once it reaches steady-state. This is an effect of the continuum model and does not contradict the fact that chloride ions are excluded from the channel given 
that $n \ll 1$. Also, we see that there is an average of approximately $4.5 \mathrm{~K}^{+}$ions in the channel during steady state. These averages were computed by numerically integrating the densities over the channel.

Figure 4 shows the steady state potential. The changes in concavity coincide with the different interior regions of the channel. We can also see that the potential varies smoothly in the baths but varies dramatically in the channel.

It is often of interest to know the current in the channel in response to a timevarying voltage signal so a useful numerical experiment is to compute this timedependent current $I(t)$ for some different voltage signals. Once the current densities are known, this is a simple matter since $I=J A$ where $A$ is the cross-sectional area of the channel or baths and $J$ is the current density defined in equation (3). The plot shown in Figure 5 was generated by calculating the current at an arbitrary bath point $(x=8)$ with a square wave voltage source defined by

$$
V(t)=\left\{\begin{aligned}
-0.1 & \text { if } 0 \leq t \leq 10 \\
0 & \text { if } t>10
\end{aligned}\right.
$$

A similar current plot, shown in Figure 6, was generated using a sinusoidal voltage signal defined by

$$
V(t)=0.1(\cos (\omega t)-1)
$$

with $\omega=4$. The plot shown in Figures 5 is exactly what one would expect for

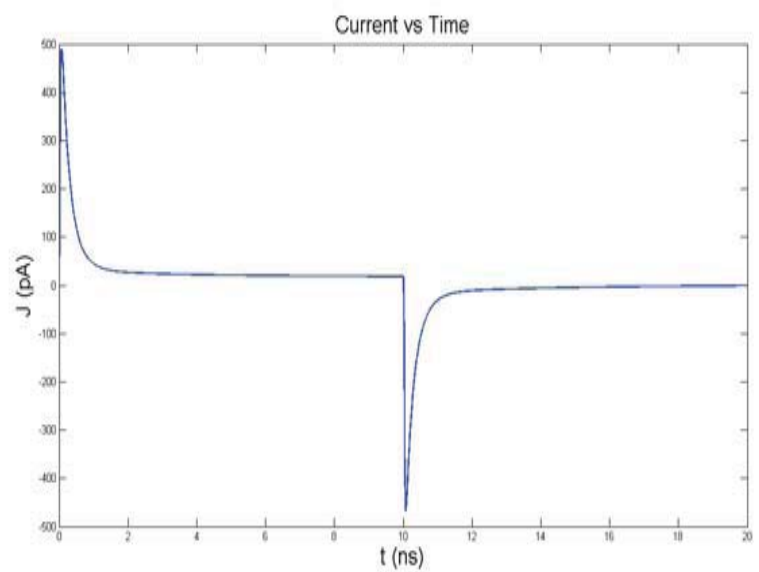

Figure 5: Current for a square wave voltage source.

a square wave voltage signal.The current is nearly zero at all times, except when 


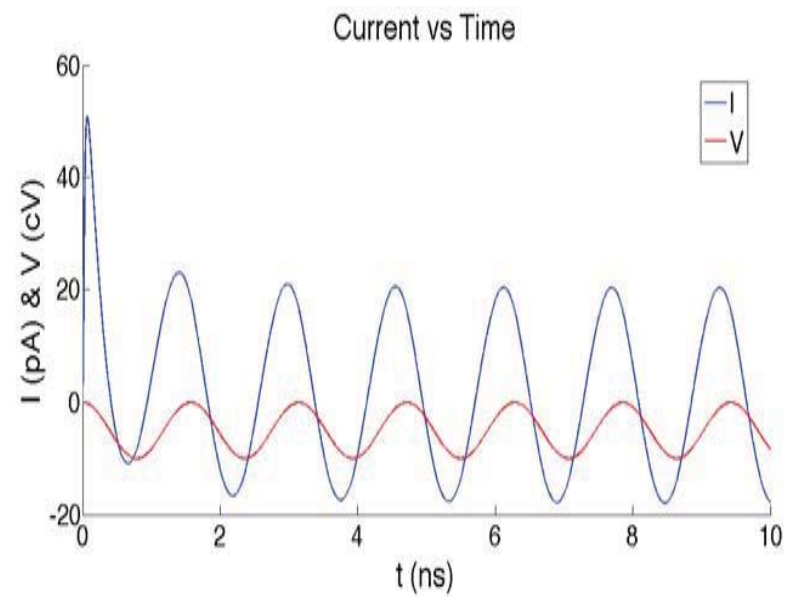

Figure 6: Current for a sinusoidal voltage source.

the voltage is turned on at $t=0 \mathrm{~ns}$ and turned off at $t=10 \mathrm{~ns}$, which causes large spikes in the current, nearly reaching $\pm 500 \mathrm{pA}$. The current quickly decays to 20 pA during the first five ns and then goes to zero once the voltage is switched off. Figure 6 shows that the current reaches steady state very quickly (about 3 ns) in response to a sinusoidal signal. Another interesting feature of Figure 6 is that the current and voltage waves are slightly out of phase, signifying a small time lag. Finally, an IV curve was generated by plotting the steady-state current for five

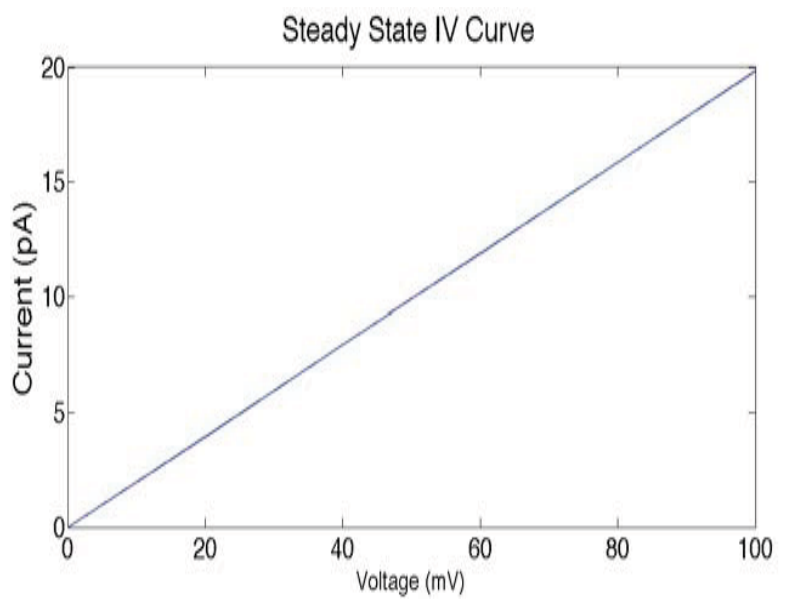

Figure 7: Current vs. Voltage. 
different voltages (see Figure 7); this in in excellent agreement with experimental data. Note the curve shown appears to be linear although experimental curves appear to be slightly super- or sub-linear (the interested reader can find these data in [1]). The super/sub-linear appearance of the experimental curve is believed to derive from the coupling to the experimental patch-clamp device. This curve also verifies the model and simulation methods since it agrees with experiment.

\section{Conclusion}

We have seen that a continuum model can serve as a useful and powerful tool in the quantitative analysis of ionic channels. Comparing the results of this paper with experimental data provides further justification of the continuum model. In addition, we were also able to make some useful predictions regarding the current that flows in response to time-varying voltage signals.

Discrete models that are more realistic require computation times that are several orders of magnitude greater than those required for the continuum model and thus are not able to simulate the channel to steady state in a practical amount of time. Although the continuum model is far from perfect, it does provide a method of approximating the behaviour of channels and running simple simulations quickly.

The 1D model explored in this paper is certainly useful but is not very realistic since the channel really has a 3-dimensional structure. However, it is not unreasonable to assume angular symmetry and solve the equations in 2D cylindrical coordinates $(r, z)$. Although the equations to be solved remain the same, the boundary conditions for the 2D problem are quite different (and much more difficult to implement) than those used in the 1D problem. The software for solving this problem is currently under development. 


\section{References}

[1] B. Hille, Ion Channels of Excitable Membranes, Sinauer Associates, Inc., Sunderland, MA, 2001.

[2] C. L. Gardner, W. Nonner, R. S. Eisenberg, "Electrodiffusion Model Simulation of Ionic Channels: 1D Simulations," Journal of Computational Electronics 3 (2004).

[3] R.E. Bank, W.M. Coughran, W. Fichtner, E.H. Grosse, D.J. Rose, and R.K. Smith, "Transient Simulation of Silicon Devices and Circuits," IEEE Transactions on Computer-Aided Design 4 (1985).

[4] R.S. Eisenberg, M.M. Klosek, and Z. Schuss, "Diffusion as a Chemical Reaction: Stochastic Trajectories Between Fixed Concentrations," Journal of Chemical Physics 102 (1995).

[5] W. Nonner, D. Chen, and R.S. Eisenberg, "Anomolous Mole Fraction Effect, Electrostatics and Binding," Biophysical Journal, 74 (1998).

[6] W. Nonner and R.S. Eisenberg, "Ion Permeation and Glutamate Residues Linked by Poisson-Nernst-Planck Theory in L-type Calcium Channels," Biophysical Journal, 75 (1998). 